\title{
Bulk viscosity of liquid noble gases
}

Cite as: J. Chem. Phys. 152, 094503 (2020); https://doi.org/10.1063/1.5142364

Submitted: 12 December 2019 . Accepted: 13 February 2020 . Published Online: 03 March 2020

René Spencer Chatwell (D), and Jadran Vrabec (D)
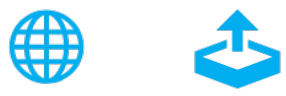

View Online

Export Citation

\section{ARTICLES YOU MAY BE INTERESTED IN}

The relation between molecular dynamics and configurational entropy in room temperature ionic liquids: Test of Adam-Gibbs model

The Journal of Chemical Physics 152, 091101 (2020); https://doi.org/10.1063/1.5140569

The hard sphere diameter of nanocrystals (nanoparticles)

The Journal of Chemical Physics 152, 094502 (2020); https://doi.org/10.1063/1.5132747

The EXP pair-potential system. IV. Isotherms, isochores, and isomorphs in the two crystalline phases

The Journal of Chemical Physics 152, 094505 (2020); https://doi.org/10.1063/1.5144871
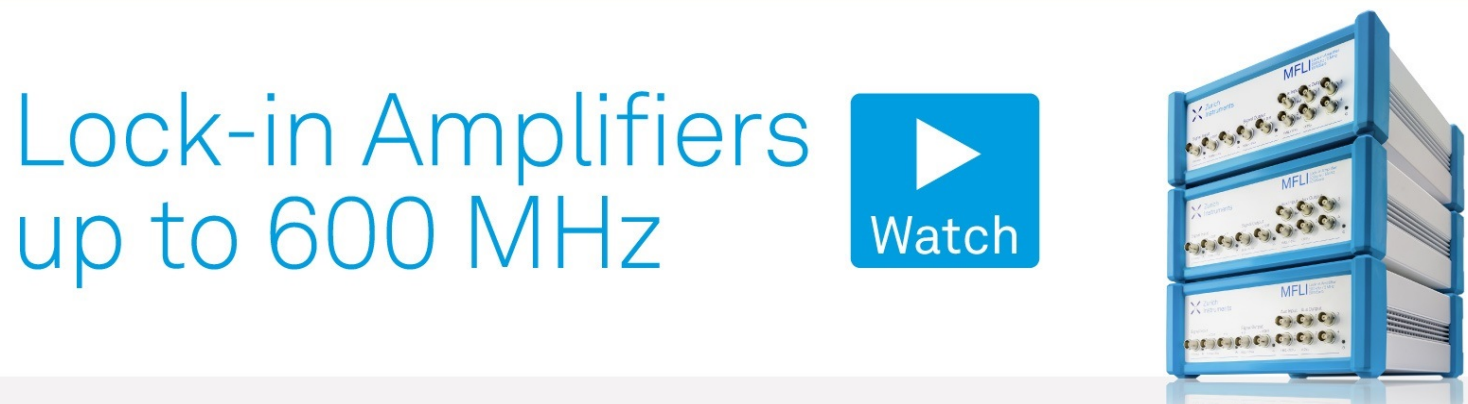

J. Chem. Phys. 152, 094503 (2020); https://doi.org/10.1063/1.5142364

152,094503 (c) 2020 Author(s) 


\title{
Bulk viscosity of liquid noble gases
}

\author{
Cite as: J. Chem. Phys. 152, 094503 (2020); doi: 10.1063/1.5142364 \\ Submitted: 12 December 2019 - Accepted: 13 February 2020 • \\ Published Online: 3 March 2020
}

René Spencer Chatwell (D) and Jadran Vrabec ${ }^{a)}$ (iD

\author{
AFFILIATIONS \\ Thermodynamics and Process Engineering, Technische Universität Berlin, 10587 Berlin, Germany
}

a) Author to whom correspondence should be addressed: vrabec@tu-berlin.de

\begin{abstract}
An equation of state for the bulk viscosity of liquid noble gases is proposed. On the basis of dedicated equilibrium molecular dynamics simulations, a multi-mode relaxation ansatz is used to obtain precise bulk viscosity data over a wide range of liquid states. From this dataset, the equation of state emerges as a two-parametric power function with both parameters showing a conspicuous saturation behavior over temperature. After passing a temperature threshold, the bulk viscosity is found to vary significantly over density, a behavior that resembles the frequency response of a one pole low-pass filter. The proposed equation of state is in good agreement with available experimental sound
\end{abstract} attenuation data.

Published under license by AIP Publishing. https://doi.org/10.1063/1.5142364

\section{INTRODUCTION}

In contrast to prevailing opinion, bulk viscous effects have widely been explored in fluid mechanics and even share a somewhat controversial history. In 1845, Stokes ${ }^{1}$ had argued geometrically that Cauchy's stress tensor field,

$$
\Pi_{i j}=\left(\mu_{b}-\frac{2}{3} \mu_{s}\right) \partial_{n} v_{n} \delta_{i j}+\mu_{s}\left(\partial_{j} v_{i}+\partial_{i} v_{j}\right),
$$

entails not only the well-known shear viscosity $\mu_{s}$ but also the bulk viscosity $\mu_{b}$, which he concurrently postulated to be zero,

$$
\mu_{b} \stackrel{!}{=} 0 \text {. }
$$

Stokes, whose proof can hardly be considered rigorous, had limited his argument to incompressible fluids and remained skeptical that his finding (2) would emerge as universally valid. For more than a century, this hypothesis was afflicted with misconceptions until the Royal Society hosted "a discussion on the first and second viscosities of fluids," ${ }^{3-18}$ reinvigorating investigations of bulk viscous effects on a wide variety of fluid mechanical problems.

Riemann's solution ${ }^{19}$ of the Euler equation ${ }^{20}$ was among the first. Retaining the bulk viscosity in the stress tensor field ${ }^{21}$ conclusively solved the problem of insensibly small wave thicknesses that were predicted ${ }^{22,23}$ in descriptions limited to shear viscosity. ${ }^{24}$ The conjectured increase in wave thickness was qualitatively confirmed in successive atomistic simulations of non-ideal liquids ${ }^{25,26}$ and in numerical investigations of rarefied gases, ${ }^{27-29}$ given that the shock structure is symmetric. ${ }^{30}$

Bulk viscous effects have long been considered mere higherorder contributions. ${ }^{31}$ However, including the bulk viscosity in the acoustic approximation ${ }^{32,33}$ straightforwardly explained the observed second-order fields associated with ultrasonic waves. The vorticity that is generated across a propagating wave's free surface induces a counter-oriented circulatory flow ${ }^{34-39}$ that is also known as acoustic streaming or quartz-wind. In hypersonic boundary layer approximations, bulk viscous effects are promoted to second-order in the pressure distribution ${ }^{40}$ and even first-order in the outer and inner flow velocities of high Reynolds number flows. $^{41}$

In fluids confined to capillaries, the bulk viscosity contributes to first-order in radial pressure and to second-order in the axial velocity. $^{42-47}$

Bulk viscous behavior was also investigated for more complex scenarios. An increased shock wave thickness affects the outer flow's adverse pressure gradient and consequently suppresses the shock induced boundary layer separation, ${ }^{48}$ while additionally the shock wave's location and strength are much more accurately predicted when bulk viscous effects are included. ${ }^{49}$ Likewise, bulk viscous damping has been observed in compressible turbulent flows. ${ }^{50} \mathrm{~A}$ non-zero bulk viscosity enhances kinetic energy dissipation while additionally inhibiting the energy transfer between translational and configurational energy, thus rendering the flow effectively incompressible. 
Each of the aforementioned investigations, however, has suffered from missing, incomplete, or unreliable bulk viscosity data and was consequently restricted to predominantly qualitative results.

\section{SOUND ATTENUATION}

Since its introduction, the bulk viscosity was closely associated with linear acoustics. Stokes had originally proposed that sound attenuation measurements could confirm his hypothesis (2) experimentally. A propagating wave's linear momentum is dissipated by a surrounding atmosphere, which leads to the exponential decay of its amplitude $A(z)$ over traveled distance $\Delta z=z_{2}-z_{1}$ and is measured by an attenuation coefficient $\alpha$,

$$
\frac{A\left(z_{2}\right)}{A\left(z_{1}\right)}=\exp \left(-\alpha_{\lambda} \frac{\Delta z}{\lambda}\right)
$$

given here per wavelength $\lambda$, i.e., $\alpha_{\lambda}=\alpha \lambda$. In a first estimate of $\alpha_{\lambda}$ for one-dimensional motion, limited to shear viscosity $\mu_{s}$, Stokes found that the attenuation factorizes into frequency $\omega=2 \pi f$ and a transport function $\xi$,

$$
\alpha_{\lambda}=\omega \xi^{\text {Stokes }}=\omega \frac{\pi}{K} \frac{4}{3} \mu_{s},
$$

where $K=\rho c^{2}$ is the fluid's low-frequency modulus of compression, $\rho$ its density, and $c$ its thermodynamic speed of sound. Kirchhoff $^{52}$ advanced the discussion by including heat conduction and established that both effects superimpose linearly in the transport function,

$$
\begin{aligned}
\alpha_{\lambda}^{\text {classical }} & =\omega\left(\xi^{\text {Stokes }}+\xi^{\text {Kirchhoff }}\right) \\
& =\omega \frac{\pi}{K}\left(\frac{4}{3} \mu_{s}+(\kappa-1) \frac{\gamma}{c_{p}}\right),
\end{aligned}
$$

where $\kappa=c_{p} / c_{v}$ is the ratio of specific heats and $\gamma$ is the thermal conductivity. The attenuation predicted by this classical theory corresponds ostensibly well with low frequency acoustic measurements.

Subsequently performed ultrasonic measurements, ${ }^{53-57}$ however, disclosed large deviations from the classical description (5) and motivated Herzfeld ${ }^{58}$ and Kneser ${ }^{59,60}$ to introduce an additional mechanism,

$$
\alpha_{\lambda}=\alpha_{\lambda}^{\text {classical }}+\alpha_{\lambda}^{\text {excess }} .
$$

Both connected classical hydrodynamics to relaxation theory and attributed the excess attenuation $\alpha_{\lambda}^{\text {excess }}$ to a time lag that occurs during the transfer of energy between the molecules' translational and internal degrees of freedom. In contrast, Tisza ${ }^{61}$ refrained from any physical interpretation and incorporated the Herzfeld mechanism into the transport function $\xi$ by force fitting the complex mechanism of relaxation into a scalar valued bulk viscosity, ${ }^{6}$

$$
\begin{aligned}
\alpha_{\lambda} & =\omega\left(\xi^{\text {Stokes }}+\xi^{\text {Kirchhoff }}+\xi^{\text {Tisza }}\right) \\
& =\omega \frac{\pi}{K}\left(\frac{4}{3} \mu_{s}+(\kappa-1) \frac{\gamma}{c_{p}}+\mu_{b}\right) .
\end{aligned}
$$

This extended theory has been validated experimentally up to moderate ultrasonic frequencies, i.e., $f \leq 280 \mathrm{MHz},{ }^{63}$ over a wide range of thermodynamic states.
In the extended vicinity of the critical point, however, an anomalously high attenuation was observed ${ }^{64-66}$ that was conclusively attributed to long-range density correlations, ${ }^{67-73}$ which are alterations of structural relaxation effects. ${ }^{74-77}$ The total attenuation partitions into the already established background part (7) and a critical contribution,

$$
\alpha_{\lambda}^{\text {total }}=\alpha_{\lambda}+\alpha_{\lambda}^{\text {critical }}
$$

Acoustic dispersion, i.e., the frequency dependent speed of sound $c(\omega)$, confines this critical attenuation to an extended critical region in the thermodynamic state space, cf. supplementary material,

$$
\alpha_{\lambda}^{\text {critical }}=\pi \frac{I(\tilde{\omega})}{J(\tilde{\omega})}\left(\left[\frac{c(\omega)}{c}\right]^{2}-1\right),
$$

where $I(\tilde{\omega}), J(\tilde{\omega})$ are improper integrals of a characteristic frequency $\tilde{\omega}$.

\section{EXPERIMENTAL DATA}

At present, the bulk viscosity is determined experimentally either by non-resonant Rayleigh-Brillouin scattering ${ }^{80-90}$ or ultrasonic attenuation measurements. ${ }^{91-121}$ Both techniques have successfully been applied to a variety of substances, yet each measurement series was restricted to selective thermodynamic states.

A substantially larger dataset, however, can be obtained for liquid noble gases by utilizing their self-similar behavior. While all available sound attenuation measurements for neon, argon, krypton, and xenon were evaluated, data subject to critical attenuation

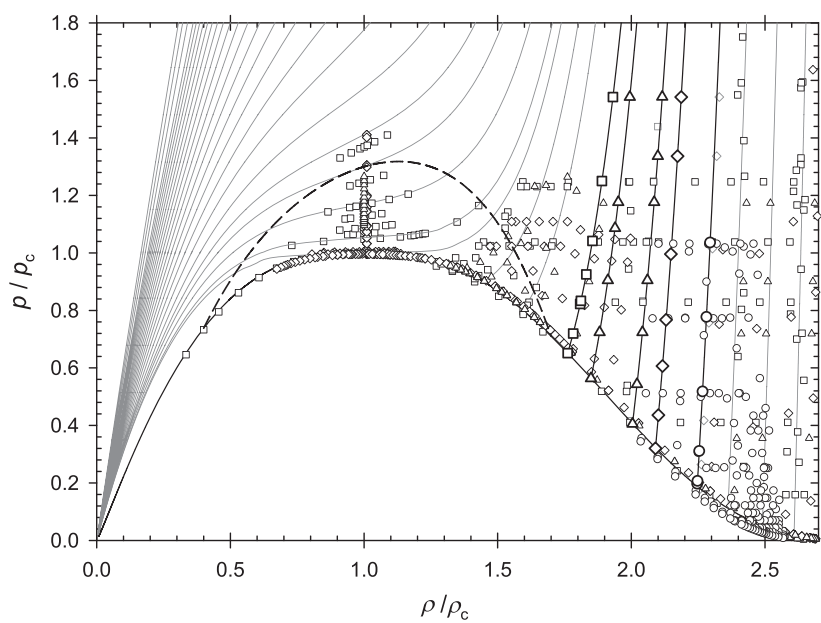

FIG. 1. Overview of thermodynamic states at which experimental sound attenuation data are available for neon ${ }^{106}$ (circles), argon ${ }^{98-101,110,112,113,115,116}$ (squares), krypton ${ }^{115-117,119}$ (triangles), and xenon ${ }^{79,115,116,118,119,122}$ (diamonds). Highlighted: Seven isotherms were selected $T / T_{c}=0.759,0.76,0.83,0.86$, $0.863,0.91$, and 0.93 , while $T / T_{\mathrm{c}}=0.759$ and 0.863 were omitted in the plot for visibility reasons, along which atomistic simulations were performed to complement and extend the dataset to higher pressures $p / p_{c} \leq 21$. All thermodynamic states were reduced with the respective fluid's critical pressure $p_{\mathrm{c}}$ and density $\rho_{\mathrm{c}}$. The extended critical region was constructed on the basis of sound dispersion measurements from the literature and is delimited by the dashed line. 
TABLE I. The following parameters for the full, i.e., untruncated Lennard-Jones potential $\sigma, \epsilon$, and atomic mass $m$ were used in the canonical transformation ${ }^{130}$ to reduce the bulk viscosity and time $t^{\star}=t \sqrt{\epsilon /\left(m \sigma^{2}\right)}$, where $k_{B}$ is Boltzmann's constant.

\begin{tabular}{lrrr}
\hline \hline & $\epsilon / k_{B}(\mathrm{~K})$ & $\sigma(\AA)$ & $m(\mathrm{u})$ \\
\hline $\mathrm{Ne}$ & 33.92 & 2.801 & 20.180 \\
$\mathrm{Ar}$ & 116.79 & 3.395 & 39.948 \\
$\mathrm{Kr}$ & 162.58 & 3.627 & 83.798 \\
$\mathrm{Xe}$ & 226.14 & 3.949 & 131.293 \\
\hline \hline
\end{tabular}

were identified and discarded, cf. Fig. 1. The bulk viscosity was calculated on the basis of the extended classical theory (7) and in contrast to previous works, more accurate thermodynamic data were available by resorting to most recent equations of state. ${ }^{123-127}$ Subsequently, $\mu_{b}$ was reduced by a canonical transformation resting on the Lennard-Jones potential, i.e., $\mu_{b}^{\star}=\mu_{b} \sigma^{2} / \sqrt{m \epsilon}$, with the required set of parameters specified in Table I.

Each contribution to bulk viscosity is afflicted by a different uncertainty. While the uncertainty of most recent thermodynamic data are well established to range from $\Delta \rho / \rho=0.005$ to $\Delta \mu_{s} / \mu_{s}$ $=0.3$ depending on substance and state, the attenuation coefficient $\alpha_{\lambda}$ has been determined by single measurements at the respective state point, and thus, only its absolute maximum errors $\Delta \alpha_{\lambda}$ have been estimated. Moreover, it is not assured that systematic measurement errors in the literature data, specifically diffraction effects, ${ }^{128}$ were properly accounted for. Consequently, the reduced bulk viscosity's uncertainty $\Delta \mu_{b}$ was determined to be linearly affected ${ }^{129}$ by its various contributions $\Delta \alpha_{\lambda}, \Delta c, \Delta \mu_{s}, \Delta \gamma, \Delta c_{v}, \Delta c_{p}$,

$$
\Delta \mu_{b}=\left|\frac{K}{\omega \pi}\right| \Delta \alpha_{\lambda}+\cdots+\left|\frac{(\kappa-1) \gamma}{c_{p}^{2}}\right| \Delta c_{p} .
$$

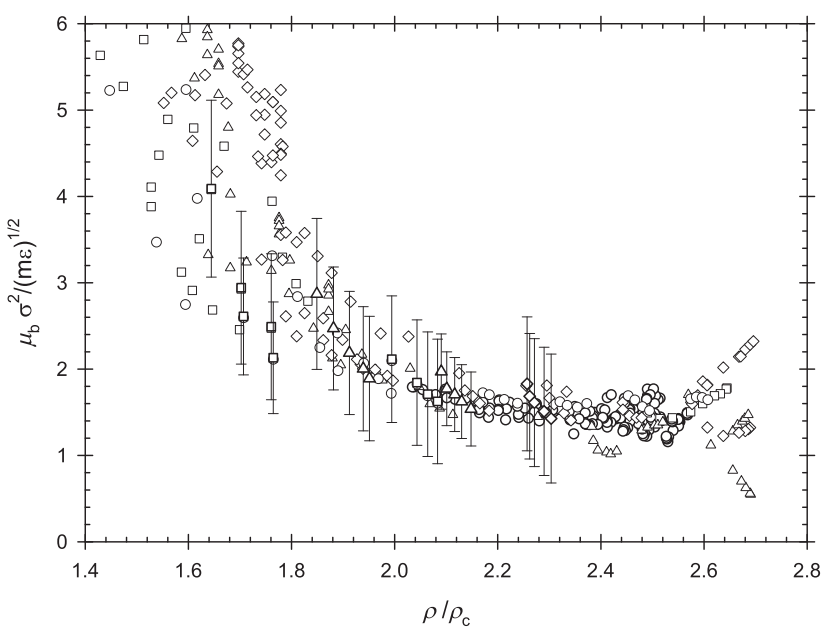

FIG. 2. Overview of bulk viscosity data calculated from the extended classical theory on the basis of experimental sound attenuation data, taken from the literature at the state points indicated in Fig. 1. Highlighted: Experimental data at the selected isotherms $T / T_{c}=0.76,0.83,0.86,0.91,0.96$ with their respective uncertainties, calculated from Eq. (10), indicating a monotonic decline of $\mu_{b}$ as a function of density.
After selecting and evaluating the experimental data, a decline of the bulk viscosity along each isotherm, i.e., from saturation line toward higher density, can qualitatively be inferred. The effect tends to increase with temperature, however, due to large errors neither the function's gradient nor the curvature can accurately be specified, cf. Fig. 2.

\section{MOLECULAR DYNAMICS SIMULATION}

To interpret and extend the experimental dataset to higher densities, additional bulk viscosity data were sampled by equilibrium molecular dynamics (EMD) simulations along seven selected isotherms $T / T_{\mathrm{c}}=0.759,0.76,0.83,0.86,0.863,0.91$, 0.93 , cf. Fig. 1. The bulk viscosity was determined microscopically by time-autocorrelation functions of local small-scale, transient pressure fluctuations ${ }^{131,132}$ that are intrinsic in any fluid under equilibrium. ${ }^{133}$ The Lennard-Jones interaction potential was used, which has demonstrated to resolve such small-scale dynamics adequately and hence successfully describes macroscopic transport in liquid noble gases. ${ }^{134-138}$

In the present work, the bulk viscosity's autocorrelation function $B_{A}$ was sampled in the microcanonical (NVE) ensemble, utilizing the fully open source program $m s 2 .{ }^{139}$ The necessary average energies $\langle E\rangle$ were determined by preceding canonical (NVT) ensemble simulations for the given pair of temperature and density. Finite size effects were minimized by placing $N=4096$ particles in cubic volumes with periodic boundary conditions and choosing a sufficiently large cutoff radius $r_{c} \geq 5.5 \sigma .^{140-142}$ The employed particle number is well chosen, as simulations containing $N=12000$ particles yielded virtually identical results, cf. supplementary material. In order to adequately resolve both the existing small-scale dynamics and also the slowly decaying pressure fluctuations, ${ }^{143}$ a reduced integrator time step $\Delta t^{\star}=5 \cdot 10^{-4}$ was specified and each autocorrelation function $B_{A}$ was sampled over a reduced time period of at least $t^{\star} \geq 14.6$.

\section{A. Relaxation ansatz}

The fluid's intrinsic small-scale pressure fluctuations have conclusively been established to relax in different modes. ${ }^{144-146}$ Each mode decays exponentially over time following a KohlrauschWilliams-Watts function. ${ }^{147,148}$ For all of the investigated state points, three superimposing relaxation modes were found to be present, leading to the relaxation model's analytical form,

$$
\begin{aligned}
B_{R}(t)= & C_{\mathrm{f}} \exp \left(-\left(\frac{t}{\delta_{\mathrm{f}}}\right)^{\beta_{\mathrm{f}}}\right)+C_{\mathrm{m}} \exp \left(-\left(\frac{t}{\delta_{\mathrm{m}}}\right)^{\beta_{\mathrm{m}}}\right) \\
& +C_{\mathrm{s}} \exp \left(-\left(\frac{t}{\delta_{\mathrm{s}}}\right)^{\beta_{\mathrm{s}}}\right) .
\end{aligned}
$$

The first term describes the fast, and the subsequent terms describe the intermediate and slow modes, respectively. The weighting factors are constraint $C_{\mathrm{f}}+C_{\mathrm{m}}+C_{\mathrm{s}}=1$, and the Kohlrausch parameters $\delta_{i}, \beta_{i}$ are a measure of relaxation time scale and distortion from the exponential function, respectively. The eight independent parameters of Eq. (11) were determined by fitting the relaxation model $B_{R}$ to the sampled autocorrelation function $B_{A}$ at each state point independently. 
Each mode's average relaxation time $\tau_{i}$ is properly defined as integral mean value of its respective contribution $B_{R, i}$ to the relaxation model, ${ }^{149}$

$$
\tau_{i}=\frac{1}{C_{i}} \lim _{t \rightarrow \infty} \int_{0}^{t} d t\left(B_{R, i}(t)\right) .
$$

As originally proposed by Maxwell, the bulk viscosity $\mu_{b}$ is proportional to the cumulative averaged relaxation time,

$$
\mu_{b}=K_{r} \sum_{i} C_{i} \tau_{i}
$$

with the proportionality constant $K_{r}$ being the fluid's relaxation modulus.

\section{RESULTS}

\section{A. Relaxation times}

The present results are exemplary discussed for states along the isotherm $T / T_{c}=0.86$ yet are qualitatively similar for all other investigated state points as disclosed in the supplementary material.

The sampled autocorrelation function $B_{A}$ partitions into three segments, with each segment being dominated by a different mode, cf. Fig. 3. The two dents in the sampled signal readily indicate the fast and intermediate mode's decay. Accompanying the latter, incipiently small scale oscillations are amplified, giving rise to substantial noise contributions in the slow mode. After reconstructing its signal by post-processing, the good agreement between the sampled autocorrelation function $B_{A}$ and the proposed relaxation model $B_{R}$ becomes apparent.

More importantly, in contrast to the sampled autocorrelation function that is plagued by noise, the employed relaxation model's time integral properly converges to a definite value, thus allowing to determine the bulk viscosity unambiguously at each state point, cf. Fig. 4.

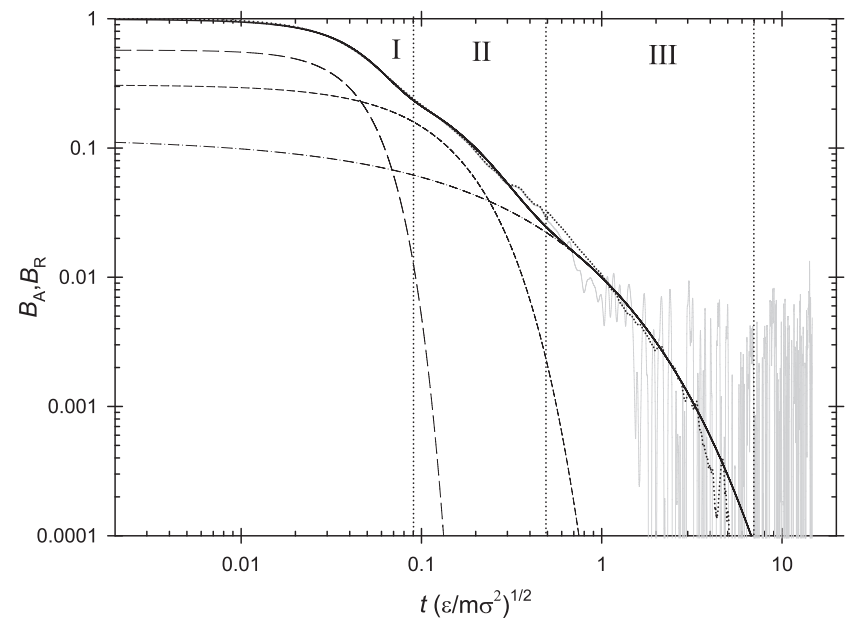

FIG. 3. Comparison of sampled autocorrelation function and relaxation model including all three relaxation modes. The gray line constitutes $B_{A}$ sampled at $T / T_{C}$ $=0.86$ and $\rho / \rho_{\mathrm{c}}=2.06$, while the solid black line represents the relaxation model (11), and its fast, intermediate, and slow modes are depicted by dashed, shortdashed, and dashed-dotted lines, respectively. The post-processed simulation signal (dotted line) is in good agreement with the relaxation model.

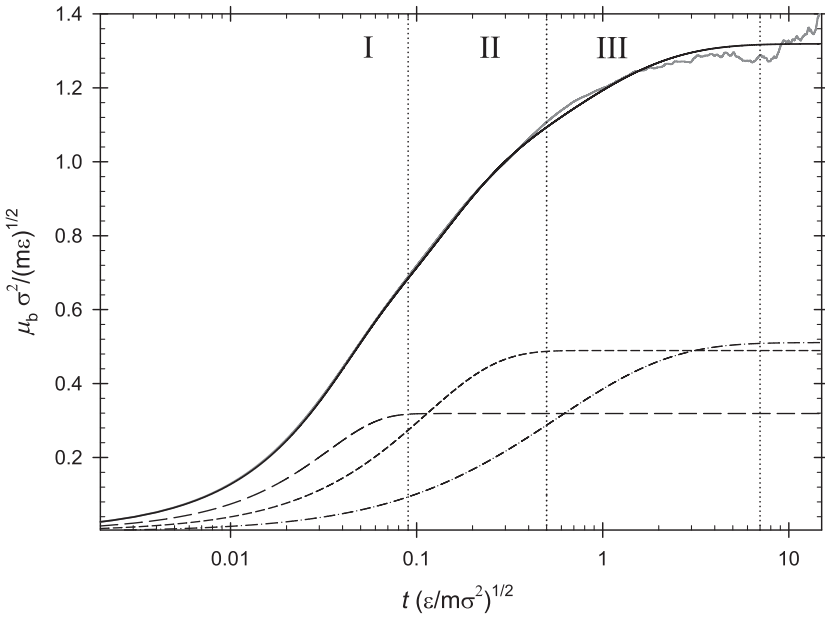

FIG. 4. Comparison of the integrated autocorrelation function $B_{A}$ with the relaxation model's (11) integral at $T / T_{\mathrm{c}}=0.86$ and $\rho / \rho_{\mathrm{c}}=2.06$. Due to noise contributions to $B_{A}$, the bulk viscosity $\mu_{b}$ is difficult to determine precisely by molecular dynamics simulation (gray line). In contrast, the proposed relaxation model (solid black line) converges toward an unambiguous value at finite times. The dashed, short-dashed, and dotted-dashed lines represent the fast, intermediate, and slow modes, respectively.

The average reduced relaxation times $\tau_{i}$ were found to decline exponentially with density for each mode and to differ roughly by one order of magnitude among the modes up to $\rho / \rho_{c} \leq 2.2$. While all relaxation modes are increasingly damped with rising density, facilitating shorter relaxation times, the slow mode is damped disproportionately, cf. Fig. 5.

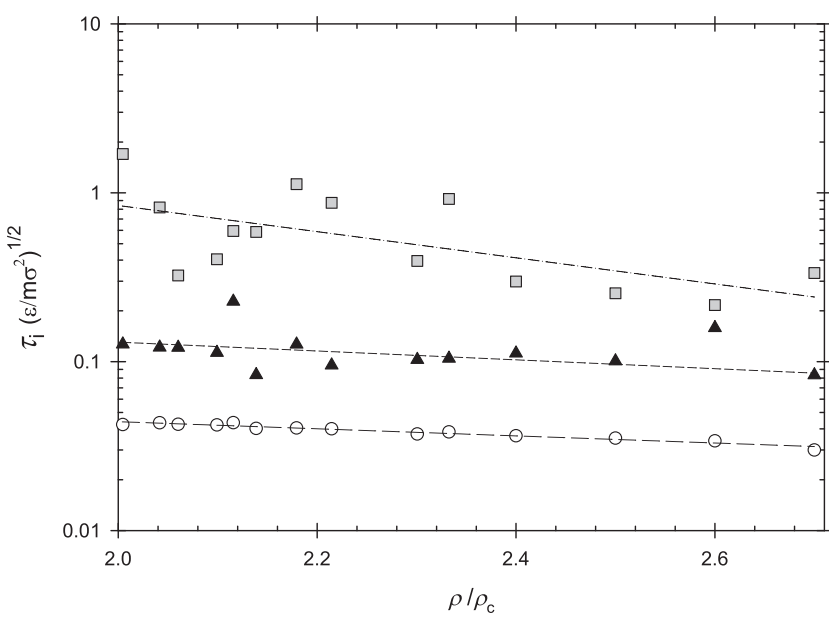

FIG. 5. Distribution of average reduced relaxation times $\tau_{i}$ for all three modes along the isotherm $T / T_{\mathrm{c}}=0.86$. While each mode is identified to relax exponentially, the slow mode is predominantly affected by increasing density. The symbols represent the relaxation times of the fast $\tau_{\mathrm{s}}$ (white circles), the intermediate $\tau_{\mathrm{m}}$ (black triangles), and the slow mode $\tau_{\mathrm{s}}$ (gray squares), respectively. 


\section{B. Equation of state}

The most recognized equation of state, of the very few that have actually been discussed in the literature, ${ }^{151,152}$ relates the bulk viscosity to a power function of density,

$$
\mu_{b} \propto \rho^{\alpha}
$$

for which special case solutions with fixed exponents $2 \alpha=-1,0,1,3$ exist. ${ }^{153-156}$ This proportionality, which was originally proposed in the context of viscous cosmological fluids, is much more universal and also applies to liquid noble gases if the observed temperature dependence is included.

After consolidating all relaxation results, a threshold temperature $T_{t} / T_{c} \sim 0.74$ emerges below which the reduced bulk viscosity indicates virtually no variation with density.

In contrast, at higher temperatures, $\mu_{b}$ increases progressively toward saturated liquid states, an effect that intensifies with rising temperature, cf. Fig. 6.
TABLE II. Best fit parameters $a_{i}, b_{i}, c_{i}$ of Eq. (16).

\begin{tabular}{lrrr}
\hline \hline & $a_{i}$ & \multicolumn{1}{c}{$b_{i}$} & $c_{i}$ \\
\hline$\alpha_{1}$ & -0.93 & 5.91 & 8.67 \\
$\alpha_{2}$ & -0.53 & -1.49 & 7.86 \\
\hline
\end{tabular}

While any physically sound equation of state must necessarily establish a unique one-to-one relation between $\mu_{b}$ and each state point, i.e., being bijective, entropy constraints additionally restrict this equation to be non-negative $e^{157,158}$ and the present results further specify it to be convex with monotonically decreasing gradient and curvature. Satisfying all conditions, a reduced two-parametric power function is proposed

$$
\mu_{b}^{\star}=\left(\frac{\rho}{\rho_{c}}-1\right)^{\alpha_{1}}+\alpha_{2}
$$

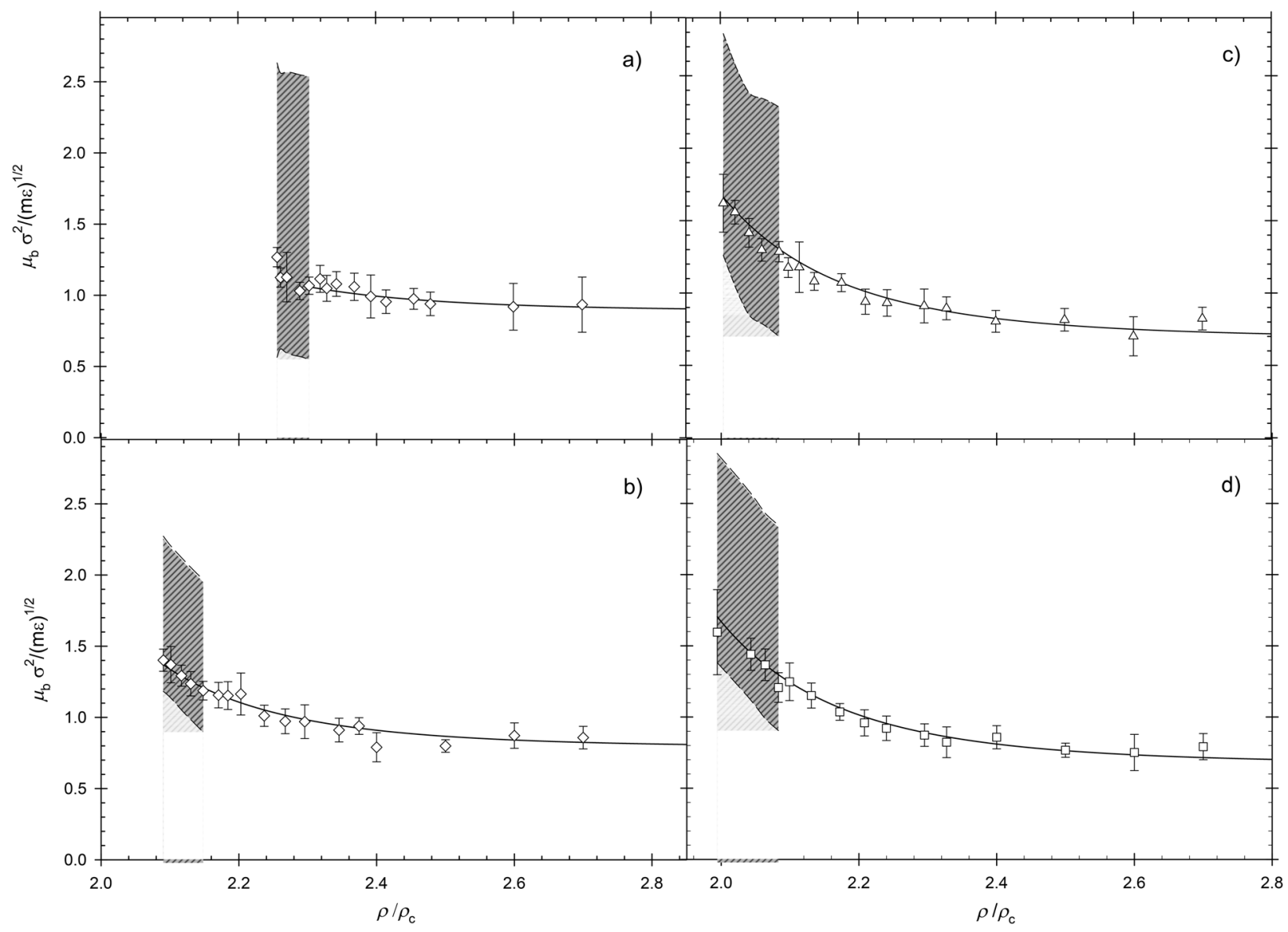

FIG. 6. Outline of bulk viscosity variations from the saturation line toward high density along four isotherms (a) $T / T_{\mathrm{c}}=0.759$, (b) $T / T_{\mathrm{c}}=0.83$, (c) $T / T_{\mathrm{c}}=0.86$, and (d) $T / T_{\mathrm{c}}$ $=0.863$. The gray shaded areas represent the experimentally determined bulk viscosity, according to Eq. (7), including its absolute maximum error, i.e., $\mu_{b} \pm \Delta \mu_{b}$, according to Eq. (10). The symbols indicate bulk viscosity data obtained by the employed relaxation ansatz (11) on the basis of the present EMD simulations. The solid line constitutes the present equation of state (15). 


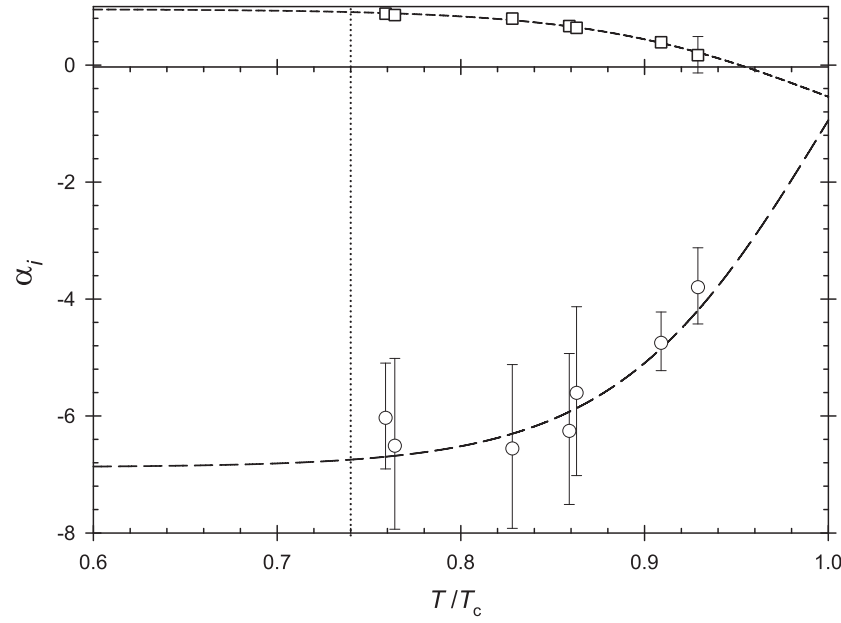

FIG. 7. Variation of parameters $\alpha_{1}$ (circles) and $\alpha_{2}$ (squares) over temperature. Above the threshold temperature $T / T_{c} \sim 0.74$ indicated by the vertical line, the bulk viscosity shows a substantial density dependence.

with parameters $\alpha_{1}, \alpha_{2}$ depending exclusively on temperature,

$$
\alpha_{i}=a_{i}+b_{i} \cdot \tanh \left(c_{i}\left(\frac{T}{T_{c}}-1\right)\right) .
$$

Both parameters, as specified in Table II, offer a salient saturation behavior that closely resembles the frequency response of a one

TABLE III. Comparison of the bulk viscosity from the present equation of state (15) with literature data that have either been obtained by molecular dynamics simulation ${ }^{140,159}$ or by theoretical calculations. ${ }^{160}$

\begin{tabular}{|c|c|c|c|c|}
\hline$T / T_{c}$ & $p / p_{c}$ & $\rho / \rho_{c}$ & $\begin{array}{c}\mu_{b} \sigma^{2} / \sqrt{m \epsilon} \\
\text { Eq. (15) }\end{array}$ & $\begin{array}{c}\mu_{b} \sigma^{2} / \sqrt{m \epsilon} \\
\text { literature }\end{array}$ \\
\hline 0.795 & 0.545 & 2.196 & 1.17 & $1.20 \pm 0.37^{140}$ \\
\hline$\ldots$ & 3.616 & 2.353 & 0.99 & $0.93 \pm 0.05$ \\
\hline$\ldots$ & 8.727 & 2.510 & 0.92 & $0.87 \pm 0.05$ \\
\hline$\ldots$ & 16.558 & 2.667 & 0.88 & $0.89 \pm 0.05$ \\
\hline 0.875 & 1.118 & 2.039 & 1.42 & $1.10 \pm 0.36$ \\
\hline$\ldots$ & 3.274 & 2.196 & 0.98 & $1.03 \pm 0.05$ \\
\hline$\ldots$ & 6.966 & 2.353 & 0.79 & $0.79 \pm 0.04$ \\
\hline 0.674 & 0.473 & 2.448 & 1.00 & $0.97 \pm 0.10^{159}$ \\
\hline 0.679 & 0.707 & $\ldots$ & 1.00 & $0.98 \pm 0.10$ \\
\hline 0.826 & 7.875 & $\ldots$ & 0.89 & $0.75 \pm 0.08$ \\
\hline 0.699 & 17.065 & 2.773 & 0.94 & $1.10 \pm 0.11$ \\
\hline 0.763 & 21.558 & $\ldots$ & 0.91 & $1.00 \pm 0.11$ \\
\hline 0.802 & 24.239 & $\ldots$ & 0.87 & $0.96 \pm 0.10$ \\
\hline 0.605 & 3.117 & 2.640 & 0.96 & $1.05^{160}$ \\
\hline 0.652 & 6.149 & $\ldots$ & 0.96 & 0.93 \\
\hline 0.667 & 7.126 & $\ldots$ & 0.96 & 1.01 \\
\hline 0.678 & 7.800 & $\ldots$ & 0.96 & 1.00 \\
\hline 0.747 & 12.086 & $\ldots$ & 0.93 & 0.86 \\
\hline 0.792 & 14.845 & $\ldots$ & 0.89 & 0.86 \\
\hline
\end{tabular}

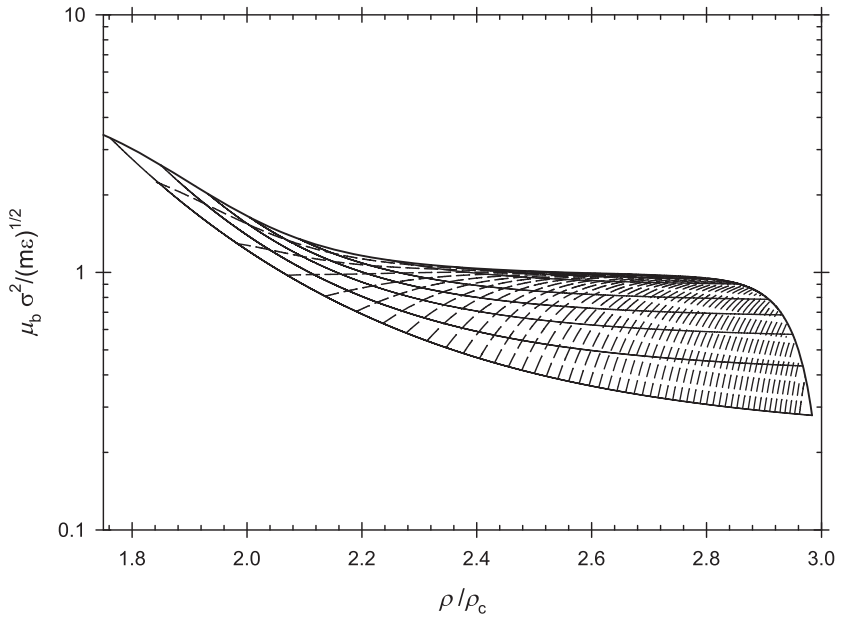

FIG. 8. Illustration of the present equation of state (15). The solid lines constitute the bulk viscosity's variation over density at constant temperature, and the dashed lines constitute its variation at constant pressure. The present results straightforwardly explain the experimental results in Fig. 2, which already suggested the bulk viscosity to be a monotonically declining function of density with increasing gradient for higher temperatures.

pole low-pass filter, cf. Fig. 7, causing the fluid to become increasingly more bulk viscous after passing the threshold temperature. This observed temperature dependence is caused by a transition from short-range order, that is present at low temperatures, ${ }^{150}$ to the long-range density correlations that are intrinsic within the extended critical region. The present equation of state is in good agreement not only with experimental sound attenuation data but also with concurrent MD simulations, cf. Table III.

\section{CONCLUSION}

An equation of state for the bulk viscosity of liquid noble gases is proposed. The bulk viscosity originates microscopically from relaxations of small-scale pressure fluctuations which were found to decay in three different modes following stretched exponential functions. The slow mode was observed to be disproportionately affected by high density and the average relaxation times $\tau_{i}$ between the modes to differ roughly by one order of magnitude. Each mode was determined on the basis of an autocorrelation function that was straightforwardly sampled by EMD simulations at the respective state point. In order to adequately resolve the slow mode, considerably long autocorrelation functions were necessary.

The equation of state emerges as a two-parametric power function with parameters depending exclusively on temperature, cf. Fig. 8. This temperature dependence is attributed to a transition from short-range order that is present at high densities to long-range density correlations that arise when approaching the extended critical region. After a threshold $T / T_{c} \sim 0.74$ is passed, the fluid becomes increasingly more bulk viscous. This effect causes sound attenuation to rise progressively with temperature, closely resembling the frequency response of a one pole low-pass filter. In addition, both bulk viscosity coefficients were observed to exhibit opposing behavior, i.e., an increase in bulk viscosity corresponds to a decline of shear viscosity and vice versa, causing the viscosities' ratio to peak 
$\mu_{b} / \mu_{s} \sim 5$ at the highest investigated temperature close to the saturation line.

\section{SUPPLEMENTARY MATERIAL}

See the supplementary material for the construction of the critical region, as well as for a full disclosure of all data associated with this manuscript.

\section{ACKNOWLEDGMENTS}

This work was carried out under the auspices of the Boltzmann-Zuse Society (BZS). All simulations were performed either on the HPC clusters OCuLUS and Noctua at the Paderborn Center for Parallel Computing $\left(\mathrm{PC}^{2}\right)$ or on the Cray CX40 system Hazel Hen at the High Performance Computing Centre Stuttgart (HLRS) with resources allocated according to Grant No. MMHBF2.

\section{REFERENCES}

${ }^{1}$ G. G. Stokes, "On the theories of the internal friction of fluids in motion, and of the equilibrium and motion of elastic solids," Cambridge Philos. Soc. 8, 287 (1845).

${ }^{2}$ A. L. Cauchy, "Recherches sur l'equilibre et le mouvement intérieur des corps solides ou fluides. Élastiques ou non élastiques," Bull. Soc. Philomath. 2, 9 (1823).

${ }^{3}$ L. Rosenhead, "Introduction. The second coefficient of viscosity: A brief review of fundamentals," Proc. R. Soc. London, Ser. A 226, 1 (1954).

${ }^{4}$ P. E. Doak, "Vorticity generated by sound," Proc. R. Soc. London, Ser. A 226, 7 (1954).

${ }^{5}$ E. G. Richardson, "Acoustic experiments relating to the coefficient of viscosity of various liquids,” Proc. R. Soc. London, Ser. A 226, 16 (1954).

${ }^{6}$ R. O. Davies, "Kinetic and thermodynamic aspects of the second coefficient of viscosity,” Proc. R. Soc. London, Ser. A 226, 24 (1954).

${ }^{7}$ G. I. Taylor, "The two coefficients of viscosity for an incompressible fluid containing air bubbles," Proc. R. Soc. London, Ser. A 226, 34 (1954).

${ }^{8}$ G. I. Taylor, "Notes on the volume viscosity of water containing bubbles," Proc, R. Soc. London, Ser. A 226, 38 (1954).

${ }^{9}$ R. O. Davies, “A note on Sir Geoffrey Taylor's paper," Proc. R. Soc. London, Ser. A 226, 39 (1954).

${ }^{10}$ H. O. Kneser, “Transport and relaxation phenomena," Proc. R. Soc. London, Ser. A 226, 40 (1954).

${ }^{11}$ J. E. Piercy and J. Lamb, “Acoustic streaming in liquids,” Proc. R. Soc. London, Ser. A 226, 43 (1954).

${ }^{12} \mathrm{~J}$. H. Andreae and J. Lamb, "Ultrasonic measurements and the second viscosity of carbon disulphide," Proc. R. Soc. London, Ser. A 226, 51 (1954).

${ }^{13}$ J. Meixner, "On the thermodynamic theory of the second viscosity," Proc. R. Soc. London, Ser. A 226, 51 (1954)

${ }^{14}$ S. M. Karim, "Experimental determination of the second viscosity," Proc. R. Soc. London, Ser. A 226, 56 (1954).

${ }^{15}$ J. G. Oldroyd, "Note on the hydrodynamic and thermodynamic pressures," Proc. R. Soc. London, Ser. A 226, 57 (1954).

${ }^{16} \mathrm{C}$. A. Truesdell, "The present status of the controversy regarding the bulk viscosity of fluids," Proc. R. Soc. London, Ser. A 226, 59 (1954).

${ }^{17}$ E. N. da C. Andrade, "Review of discussion," Proc. R. Soc. London, Ser. A 226, 65 (1954).

${ }^{18}$ R. O. Davies and L. Rosenhead, "The two viscosities of fluids," Nature 173, 1209 (1954).

${ }^{19}$ B. Riemann, "Über die Fortpflanzung ebenener Luftwellen von endlicher Schwingungsbreite," in Collected Works of Bernhard Riemann, edited by H. Weber (Dover, 1953), p. 157.

${ }^{20}$ L. Euler, "Principes généraux du mouvement des fluides," Mém. Acad. Sci. Berlin 11, 274 (1755).
${ }^{21}$ D. Gilbarg and D. Paolucci, "The structure of shock waves in the continuum theory of fluids," J. Ration. Mech. Anal. 2, 617 (1953), see https://www.jstor.org/stable/24900350.

${ }^{22}$ R. Becker, "Stoßwelle und Detonation," Z. Phys. 8, 321 (1921).

${ }^{23} \mathrm{H}$. Grad, "The profile of a steady shock wave," Commun. Pure Appl. Math. 5, 257 (1952).

${ }^{24}$ W. Tollmien, H. Schlichting, H. Görtler, and F. W. Riegels, "Zur Theorie des Verdichtungsstoßes," in Ludwig Prandtl Gesammelte Abhandlungen, edited by F. W. Riegels (Springer, Berlin, 1961).

${ }^{25}$ W. G. Hoover, "Structure of a shock-wave front in a liquid," Phys. Rev. Lett. 42 1531 (1979).

${ }^{26}$ B. L. Holian, W. G. Hoover, B. Moran, and G. K. Straub, "Shock-wave structure via nonequilibrium molecular dynamics and Navier-Stokes continuum mechanics," Phys. Rev. A 22, 2798 (1980).

${ }^{27}$ G. Emanuel and B. M. Argrow, "Linear dependence of the shock wave thickness on bulk viscosity," Phys. Fluids 6, 3203 (1994).

${ }^{28}$ T. G. Elizarova, A. A. Khokhlov, and S. Montero, "Numerical simulation of shock wave structure in nitrogen," Phys. Fluids 19, 068102 (2007).

${ }^{29}$ A. V. Chikitkin, B. V. Rogov, G. A. Tirsky, and S. V. Utyuzhnikov, "Effect of bulk viscosity in supersonic flow past spacecraft,” Appl. Numer. Math. 93, 47 (2015).

${ }^{30}$ S. Taniguchi, T. Arima, T. Ruggeri, and M. Sugiyama, "Overshoot of the nonequilibrium temperature in the shock wave structure of a rarefied polyatomic gas subject to the dynamic pressure," Int. J. Nonlinear Mech. 79, 66 (2016).

${ }^{31}$ M. van Dyke, "Second-order compressible boundary layer theory with application to blunt bodies in hypersonic flow," in Hypersonic Flow Research, edited by F. R. Riddell (Academic Press, 1962), p. 37.

${ }^{32}$ P. M. Morse and K. U. Ingard, Theoretical Acoustics (Princeton University Press, 1986).

${ }^{33}$ M. Trusler, Physical Acoustics and Metrology of Fluids (CRC Press, 1991).

${ }^{34}$ C. Eckart, "Vortices and streams caused by sound waves," Phys. Rev. 73, 68 (1948).

${ }^{35}$ L. N. Liebermann, “Second viscosity of liquids,” Phys. Rev. 75, 1415 (1949).

${ }^{36}$ J. J. Markham, "Second-order acoustic fields: Streaming with viscosity and relaxation,” Phys. Rev. 86, 497 (1952).

${ }^{37} \mathrm{P}$. J. Westervelt, "The theory of steady rotational flow generated by a sound field," J. Acoust. Soc. Am. 25, 60 (1953).

${ }^{38}$ W. L. Nyborg, "Acoustic streaming due to attenuated plane waves," J. Acoust. Soc. Am. 25, 68 (1953).

${ }^{39}$ J. Lighthill, “Acoustic streaming,” J. Sound Vib. 61, 391 (1978).

${ }^{40} \mathrm{G}$. Emanuel, "Effect of bulk viscosity on a hypersonic boundary layer," Phys. Fluids 4, 491 (1992).

${ }^{41}$ M. S. Cramer and F. Bahmani, "Effect of large bulk viscosity on large-Reynoldsnumber flows," J. Fluid Mech. 751, 142 (2014).

${ }^{42}$ H. R. van den Berg, C. A. ten Seldam, and P. S. van der Gulik, "Compressible laminar flow in a capillary," J. Fluid Mech. 246, 1 (1993).

${ }^{43}$ J. C. Harley, Y. Huang, H. H. Bau, and J. N. Zemel, "Gas flow in microchannels," J. Fluid Mech. 284, 257 (1995).

${ }^{44}$ Y. Zohar, S. Y. K. Lee, W. Y. Lee, L. Jiang, and P. Tong, "Subsonic gas flow in a straight and uniform microchannel," J. Fluid Mech. 472, 125 (2002).

${ }^{45}$ D. C. Venerus, "Laminar capillary flow of compressible viscous fluids," J. Fluid Mech. 555, 59 (2006).

${ }^{46}$ E. G. Taliadorou, M. Neophytou, and G. C. Georgiou, "Perturbation solutions of Poiseuille flows of weakly compressible Newtonian liquids," J. Non-Newtonian Fluid Mech. 163, 25 (2009).

${ }^{47}$ D. C. Venerus and D. J. Bugajsky, "Compressible laminar flow in a channel," Phys. Fluids 22, 046101 (2010).

${ }^{48} \mathrm{~F}$. Bahmani and M. Cramer, "Suppression of large shock-induced separation in fluids having large bulk viscosities," J. Fluid Mech. 756, R2 (2014).

${ }^{49} \mathrm{~S}$. Bhola and T. K. Sengupta, "Roles of bulk viscosity on transonic shockwave/boundary layer interactions," Phys. Fluids 31, 096101 (2019).

${ }^{50}$ S. Chen, X. Wang, J. Wang, M. Wan, H. Li, and S. Chen, "Effects of bulk viscosity on compressible homogeneous turbulence," Phys. Fluids 31, 085115 (2019).

${ }^{51} \mathrm{~S}$. Pan and E. Johnsen, "The role of bulk viscosity on the decay of compressible, homogeneous, isotropic turbulence," J. Fluid Mech. 833, 717 (2017). 
${ }^{52}$ G. Kirchhoff, "Über den Einfluß der Wärmeleitung in einem Gas auf die Schallbewegung," Ann. Phys. 210, 177 (1868).

${ }^{53}$ N. Neklepajev, "Über die Absorption kurzer akustischer Wellen in der Luft," Ann. Phys. 340, 175 (1911).

${ }^{54}$ T. P. Abello, "Absorption of ultrasonic waves by various gases," Phys. Rev. 31, 1083 (1928).

${ }^{55} \mathrm{~W}$. H. Pielemeier, "The Pierce acoustic interferometer as an instrument for the determination of velocity and absorption," Phys. Rev. 34, 1184 (1929).

${ }^{56} \mathrm{E}$. Großmann, "Schallabsorptionsmessung in Gasen bei hohen Frequenzen," Ann. Phys. 405, 681 (1932).

${ }^{57}$ R. W. Curtis, "An experimental determination of ultrasonic absorption an reflexion coefficients in air and in carbon dioxide," Phys. Rev. 46, 811 (1934).

${ }^{58} \mathrm{~K}$. F. Herzfeld and F. O. Rice, "Dispersion and absorption of high frequency sound waves," Phys. Rev. 31, 691 (1928).

${ }^{59}$ H. O. Kneser, "Schallabsorption in mehratomigen Gasen," Ann. Phys. 408, 337 (1933).

${ }^{60}$ H. O. Kneser, "Schallabsorption und -dispersion in Flüssigkeiten," Ann. Phys. 424, 277 (1938).

${ }^{61}$ L. Tisza, "Supersonic absorption and Stokes' viscosity relation," Phys. Rev. 61, 531 (1942).

${ }^{62}$ W. E. Meador, G. A. Miner, and L. W. Townsend, "Bulk viscosity as a relaxation parameter: Fact or fiction?," Phys. Fluids 8, 258 (1996).

${ }^{63}$ R. A. Rapuano, "Ultrasonic absorption from 75 to $280 \mathrm{Mc} / \mathrm{sec}$," Phys. Rev. 72, 78 (1947).

${ }^{64}$ W. G. Schneider, "Sound velocity and sound absorption in the critical region," Can. J. Chem. 29, 243 (1951).

${ }^{65}$ A. G. Chynoweth and W. G. Schneider, "Ultrasonic propagation in xenon in the region of its critical temperature," J. Chem. Phys. 20, 1777 (1952).

${ }^{66}$ H. D. Parbrook and E. G. Richardson, "Propagation of ultrasonic waves in vapours near the critical point," Proc. Phys. Soc., Sect. B 65, 437 (1952).

${ }^{67}$ M. Fixman, "Ultasonic attenuation in the critical region," J. Chem. Phys. 33, 1363 (1960).

${ }^{68}$ W. Botch and M. Fixman, "Sound absorption in gases in the critical region," J. Chem. Phys. 42, 199 (1965).

${ }^{69}$ M. Fixman, "Transport coefficients in the gas critical region," J. Chem. Phys. 47, 2808 (1967)

${ }^{70} \mathrm{~K}$. Kawasaki, "Correlation-function approach to the transport coefficients near the critical point. I," Phys. Rev. 150, 291 (1966).

${ }^{71} \mathrm{~K}$. Kawasaki, "Sound attenuation and dispersion near the liquid-gas critical point,” Phys. Rev. A 1, 1750 (1970).

${ }^{72}$ L. P. Kadanoff, W. Götze, D. Hamblen, R. Hecht, E. A. S. Lewis, V. V. Palciauskas, M. Rayl, and J. Swift, "Static phenomena near critical points: Theory and experiment," Rev. Mod. Phys. 39, 395 (1967).

${ }^{73}$ L. P. Kadanoff and J. Swift, "Transport coefficients near the liquid-gas critical point," Phys. Rev. 166, 89 (1968).

${ }^{74} \mathrm{~L}$. Hall, "The origin of excess ultrasonic absorption in water," Phys. Rev. 71, 318 (1947).

${ }^{75} \mathrm{~L}$. Hall, "The origin of ultrasonic absorption in water," Phys. Rev. 73, 775 (1948).

${ }^{76} \mathrm{D}$. Sette, "Ultrasonic absorption in liquid mixtures and structural effects," J. Chem. Phys. 21, 558 (1953).

${ }^{77}$ K. F. Herzfeld and T. A. Litovitz, Absorption and Dispersion of Ultrasonic Waves (Academic Press, 1959).

${ }^{78}$ C. W. Garland, D. Eden, and L. Mistura, "Critical sound absorption in xenon," Phys. Rev. Lett. 25, 1161 (1970).

${ }^{79}$ P. E. Mueller, D. Eden, C. W. Garland, and R. C. Williamson, "Ultrasonic attenuation and dispersion in xenon near its critical point," Phys. Rev. A 6, 2272 (1972).

${ }^{80}$ P. A. Fleury and J.-P. Boon, "Brillouin scattering in simple liquids: Argon and neon," Phys. Rev. 186, 244 (1969).

${ }^{81}$ A. S. Pine, "Velocity and attenuation of hypersonic waves in liquid nitrogen," J. Chem. Phys. 51, 5171 (1969).

${ }^{82}$ B. Y. Baharudin, D. A. Jackson, P. E. Schoen, and J. Rouch, "Bulk viscosity of liquid argon, krypton and xenon," Phys. Lett. A 51, 409 (1975).
${ }^{83}$ X. Pan, M. N. Shneider, and R. B. Miles, "Coherent Rayleigh-Brillouin scattering," Phys. Rev. Lett. 89, 183001 (2002).

${ }^{84}$ J. Xu, X. Ren, W. Gong, R. Dai, and D. Liu, "Measurement of the bulk viscosity of liquid by Brillouin scattering," Appl. Opt. 42, 6704 (2003).

${ }^{85}$ X. Pan, M. N. Shneider, and R. B. Miles, "Power spectrum of coherent RayleighBrillouin scattering in carbon dioxide," Phys. Rev. A 71, 045801 (2005).

${ }^{86}$ A. S. Meijer, A. S. de Wijn, M. F. E. Peters, N. J. Dam, and W. van de Water, "Coherent Rayleigh-Brillouin scattering measurements of bulk viscosity of polar and nonpolar gases, and kinetic theory," J. Chem. Phys. 133, 164315 (2010).

${ }^{87} \mathrm{X}$. He, H. Wei, J. Shi, J. Liu, S. Li, W. Chen, and X. Mo, "Experimental measurement of bulk viscosity of water based on stimulated Brillouin scattering," Opt. Commun. 285, 4120 (2012).

${ }^{88} \mathrm{Z}$. Gu and W. Ubachs, "A systematic study of Rayleigh-Brillouin scattering in air, $\mathrm{N}_{2}$, and $\mathrm{O}_{2}$ gases," J. Chem. Phys. 141, 104320 (2014).

${ }^{89} \mathrm{Z}$. Gu, W. Ubachs, and W. van de Water, "Rayleigh-Brillouin scattering of carbon dioxide," Opt. Lett. 39, 3301 (2014).

${ }^{90}$ Y. Ma, H. Li, Z. Gu, W. Ubachs, Y. Yu, J. Huang, B. Zhou, Y. Wand, and K. Liang, "Analysis of Rayleigh-Brillouin spectral profiles and Brillouin shifts in nitrogen gas and air," Opt. Express 22, 2092 (2014).

${ }^{91}$ G. W. Pierce, "Piezoelectric crystal oscillators applied to the precision measurement of the velocity of sound in air and $\mathrm{CO}_{2}$ at high frequency," Proc. Am. Acad. Arts Sci. 60, 271 (1925).

${ }^{92}$ M. Kohler, "Schallabsorption in Mischungen einatomiger Gase," Ann. Phys. 431, 209 (1941).

${ }^{93}$ J. M. M. Pinkerton, "The absorption of ultrasonic waves in liquids and its relation to molecular constitution," Proc. Phys. Soc., Sect. B 62, 129 (1949).

${ }^{94} \mathrm{M}$. Pancholy, "Temperature variation of velocity and absorption coefficient of ultrasonic waves in heavy water," J. Acoust. Soc. Am. 25, 1003 (1953).

${ }^{95} \mathrm{~T}$. A. Litovitz and E. H. Carnevale, "Effect of pressure on sound propagation in water," J. Appl. Phys. 26, 816 (1955).

${ }^{96} \mathrm{~W}$. Tempest and H. D. Parbrook, "The absorption of sound in argon, nitrogen and oxygen," Acustica 7, 354 (1957).

${ }^{97}$ D. G. Naugle and C. F. Squire, "Ultrasonic attenuation in liquid argon," J. Chem. Phys. 42, 3725 (1965).

${ }^{98}$ D. G. Naugle, "Excess ultrasonic attenuation and intrinsic volume viscosity in liquid argon," J. Chem. Phys. 44, 741 (1966).

${ }^{99}$ D. G. Naugle, J. H. Lunsford, and J. R. Singer, "Volume viscosity in liquid argon at high pressure," J. Chem. Phys. 45, 4669 (1966).

${ }^{100}$ D. S. Swyt, J. F. Havlice, and E. F. Carome, "Ultrasonic absorption in liquid argon," J. Chem. Phys. 47, 1199 (1967).

${ }^{101}$ W. M. Madigosky, "Density dependence of bulk viscosity in argon," J. Chem. Phys. 46, 4441 (1967).

${ }^{102} \mathrm{~J}$. R. Singer and J. H. Lunsford, "Ultrasonic attenuation and volume viscosity in liquid nitrogen," J. Chem. Phys. 47, 811 (1967).

${ }^{103} \mathrm{~J}$. R. Singer, "Excess ultrasonic attenuation and volume viscosity in liquid methane," J. Chem. Phys. 51, 4729 (1969).

${ }^{104}$ A. E. Victor and R. T. Beyer, "Ultrasonic absorption in liquid oxygen and nitrogen," J. Chem. Phys. 52, 1573 (1970).

${ }^{105} \mathrm{~J}$. Allegra, S. Hawley, and G. Holton, "Pressure dependence of the ultrasonic absorption in toluene and hexane," J. Acoust. Soc. Am. 47, 144 (1970).

${ }^{106}$ E. V. Larson, D. G. Naugle, and T. W. Aldair, "Ultrasonic velocity and attenuation in liquid neon," J. Chem. Phys. 54, 2429 (1971).

${ }^{107}$ S. K. Kor, O. N. Awasthi, G. Rai, and S. C. Deorani, "Structural absorption of ultrasonic waves in methanol," Phys. Rev. A 3, 390 (1971).

${ }^{108}$ S. K. Kor, S. C. Deorani, and B. K. Singh, "Origin of ultrasonic absorption in methanol," Phys. Rev. A 3, 1780 (1971).

${ }^{109}$ S. K. Kor, S. C. Deorani, B. K. Singh, R. Prasad, and U. Tandon, "Ultrasonic study of structural relaxation in ethanol," Phys. Rev. A 4, 1299 (1971).

${ }^{110} \mathrm{~J}$. A. Cowan and R. N. Ball, "Temperature dependence of bulk viscosity in liquid argon," Can. J. Phys. 50, 1881 (1972).

${ }^{111}$ G. Rai, B. K. Singh, and O. N. Awasthi, "Structural absorption of ultrasonic waves in associated liquids," Phys. Rev. A 5, 918 (1972).

${ }^{112}$ J. A. Cowan and P. W. Ward, "Ultrasonic attenuation of the bulk viscosity of liquid argon near the critical point,” Can. J. Phys. 51, 2219 (1973). 
${ }^{113}$ P. W. Ward, J. A. Cowan, and R. K. Pathria, "Critical attenuation of ultrasound in argon," Can. J. Phys. 53, 29 (1975).

${ }^{114}$ G. J. Prangsma, A. J. Alberga, and J. J. M. Beenakker, "Ultrasonic determination of the volume viscosity of $\mathrm{N}_{2}, \mathrm{CO}, \mathrm{CH}_{4}$ and $\mathrm{CD}_{4}$ between 77 and $300 \mathrm{~K}$," Physica 64, 278 (1973).

${ }^{115}$ S. A. Mikhailenko, B. G. Dudar, and V. A. Shmidt, "Volume viscosity and relaxation times in monoatomic classical fluids," Fiz. Nizk. Temp. 1, 224 (1975).

${ }^{116}$ P. Malbrunot, A. Boyer, E. Charles, and H. Abachi, "Experimental bulk viscosities of argon, krypton, and xenon near their triple point," Phys. Rev. A 27, 1523 (1983).

${ }^{117}$ J. A. Cowan and R. N. Ball, "Ultrasonic attenuation and bulk viscosity in liquid krypton," Can. J. Phys. 58, 74 (1980).

${ }^{118}$ J. A. Cowan and J. W. Leech, "Ultrasonic attenuation and bulk viscosity of liquid xenon," Can. J. Phys. 59, 1280 (1981).

${ }^{119}$ J. A. Cowan and J. W. Leech, "Critical region ultrasonic attenuation in the condensed inert gases," Can. J. Phys. 61, 895 (1983).

${ }^{120}$ A. S. Dukhin and P. J. Goetz, "Bulk viscosity and compressibility measurement using acoustic spectroscopy," J. Chem. Phys. 130, 124519 (2009).

${ }^{121}$ M. J. Holmes, N. G. Paker, and M. J. W. Povey, "Temperature dependence of bulk viscosity in water using acoustic spectroscopy," J. Phys.: Conf. Ser. 269, 012011 (2011).

${ }^{122}$ J. Thoen and C. W. Garland, "Sound absorption and dispersion as a function of density near the critical point of xenon," Phys. Rev. A 10, 1311 (1974).

${ }^{123}$ C. Tegeler, R. Span, and W. Wagner, "A new equation of state for argon covering the fluid region for temperatures from the melting line to $700 \mathrm{~K}$ at pressures up to $1000 \mathrm{MPa}$," J. Phys. Chem. Ref. Data 28, 779 (1999).

${ }^{124} \mathrm{E}$. W. Lemmon and R. T. Jacobsen, "Viscosity and thermal conductivity equations for nitrogen, oxygen, argon and air," Int. J. Thermophys. 25, 21 (2004).

${ }^{125} \mathrm{E}$. W. Lemmon and R. Span, "Short fundamental equations of state for 20 industrial fluids,” J. Chem. Eng. Data 51, 785 (2006).

${ }^{126}$ M. L. Huber, "Models for viscosity, thermal conductivity, and surface tension of selected pure fluids as implemented in REFPROP v10.0," Technical Report No. 8209, National Institute for Standards and Technology, 2014.

${ }^{127}$ R. Katti, R. T. Jacobsen, R. B. Stewart, and M. Jahangiri, "Thermodynamic properties of neon for temperatures from the triple point to $700 \mathrm{~K}$ at pressures to $700 \mathrm{MPa}$," in Advances in Cryogenic Engineering, edited by R. W. Fast (Springer, 1986), pp. 1189-1197.

${ }^{128}$ L. Claes, L. M. Hülskämper, E. Baumhögger, N. Feldmann, R. S. Chatwell, J. Vrabec, and B. Henning, "Acoustic absoprtion measurement for the determination of the bulk viscosity of pure fluids," TM - Tech. Mess. 86, 2-6 (2019).

${ }^{129}$ J. R. Taylor, Introduction to Error Analysis: The Study of Uncertainties in Physical Measurements, 2nd ed. (University Science Books, 1997).

${ }^{130}$ G. Rutkai, M. Thol, R. Span, and J. Vrabec, "How well does the Lennard-Jones potential represent the thermodynamic properties of noble gases?," Mol. Phys. 115, 1104 (2017).

${ }^{131}$ M. S. Green, "Markoff random process and the statistical mechanics of timedependent phenomena. II. Irreversible processes in fluids," J. Chem. Phys. 22, 398 (1954).

${ }^{132}$ R. Kubo, "Statistical-mechanical theory of irreversible processes. I. General theory of simple applications to magnetic and conductive problems," J. Phys. Soc. Jpn. 12, 570 (1957).

${ }^{133}$ R. Kubo, "The fluctuation-dissipation theorem," Rep. Prog. Phys. 29, 255 (1966).

${ }^{134}$ L. Verlet, "Computer "experiments" on classical fluids. I. Properties of Lennard-Jones molecules," Phys. Rev. 159, 98 (1967).

${ }^{135}$ D. Levesque and L. Verlet, "Computer "experiments" on classical fluids. III. Time-dependent self-correlation functions," Phys. Rev. A 2, 2514 (1970).

${ }^{136}$ D. Levesque, L. Verlet, and J. Kürkijarvi, "Computer "experiments" on classical fluids. IV. Transport properties and time-correlation functions of the LennardJones liquid near its triple point," Phys. Rev. A 7, 1690 (1973).
${ }^{137} \mathrm{~S}$. V. Lishchuk, "Role of three-body interactions in formation of bulk viscosity in liquid argon," J. Chem. Phys. 136, 164501 (2012).

${ }^{138}$ F. Jaeger, O. K. Matar, and E. A. Müller, "Bulk viscosity of molecular liquids," J. Chem. Phys. 148, 174504 (2018).

${ }^{139}$ G. Rutkai, A. Köster, G. Guevara-Carrion, T. Janzen, M. Schappals, C. W. Glass, M. Bernreuther, A. Wafai, S. Stephan, M. Kohns, S. Reiser, S. Deublein, M. T. Horsch, H. Hasse, and J. Vrabec, "ms2: A molecular simulation tool for thermodynamic properties, release 3.0," Comput. Phys. Commun. 221, 343 (2017)

${ }^{140} \mathrm{~K}$. Meier, A. Laesecke, and S. Kabelac, "Transport coefficients of the LennardJones model fluid. III. Bulk viscosity,” J. Chem. Phys. 122, 014513 (2005).

${ }^{141}$ V. G. Baidakov and S. P. Protsenko, "Metastable Lennard-Jones fluids. III. Bulk viscosity," J. Chem. Phys. 141, 114503 (2014).

${ }^{142}$ A. Zaragoza, M. A. Gonzales, L. Joly, I. López-Montero, M. A. Canales, A. L. Benavides, and C. Valeriani, "Molecular dynamics study of nanoconfined TIP4P/2005 water: How confinement and temperature affect diffusion and viscosity," Phys. Chem. Chem. Phys. 21, 13653 (2019).

${ }^{143}$ G. S. Fanourgakis, J. S. Medina, and R. Prosmiti, "Determining the bulk viscosity of rigid water models," J. Phys. Chem. A 116, 2564 (2012).

${ }^{144}$ G.-J. Guo, Y.-G. Zhang, K. Refson, and Y.-J. Zhao, "Viscosity and stress autocorrelation function in supercooled water: A molecular dynamics study," Mol. Phys. 100, 2617 (2002).

${ }^{145}$ G. Delgado-Barrio, P. Villareal, G. Winter, J. S. Medina, B. González, J. V. Alemán, J. L. Gomez, P. Sangrá, J. J. Santana, and M. E. Torres, "Viscosity of liquid water via equilibrium molecular dynamics simulations," in Frontiers in Quantum Systems in Chemistry and Physics, edited by S. Wilson, P. J. Grout, G. Delgado-Barrio, and P. Piecuch (Springer, 2008), pp. 351-361.

${ }^{146}$ J. S. Medina, R. Prosmiti, P. Villarreal, G. Delgado-Barrio, G. Winter, B. González, J. V. Alemán, and C. Collado, "Molecular dynamics simulations of rigid and flexible water models: Temperature dependence of viscosity," Chem. Phys. 388, 9 (2011).

${ }^{147}$ R. Kohlrausch, "Theorie des elektrischen Rückstandes in der Leidner Flasche," Ann. Phys. 167, 179 (1854).

${ }^{148} \mathrm{G}$. Williams and D. C. Watts, "Non-symmetrical dielectric relaxation behaviour arising from a simple empirical decay function," Trans. Faraday Soc. 66, 80 (1970).

${ }^{149}$ R. D. Mountain and R. Zwanig, "Shear relaxation times of simple fluids," J. Chem. Phys. 44, 2777 (1966).

${ }^{150}$ T. A. Litovitz and C. M. Davis, "Structural and shear relaxation in liquids," in Peroperties of Gases, Liquids and Solutions, Physical Acoustics, edited by W. P. Mason (Academic Press, 1965), Vol. 2, pp. 281-349.

${ }^{151}$ G. L. Murphy, "Big-bang model without singularities," Phys. Rev. D 8, 4231 (1973).

${ }^{152}$ V. A. Belinskii and I. M. Khalatnikov, "Influence of viscosity on the character of cosmological evolution,” Zh. Eksp. Theor. Fiz. 69, 401 (1975).

${ }^{153}$ I. Brevik, E. Elizalde, S. Nojiri, and S. D. Odintsov, "Viscous little rip cosmology," Phys. Rev. D 84, 103508 (2011).

${ }^{154}$ R. Colistete, J. C. Fabris, J. Tossa, and W. Zimdahl, "Bulk viscous cosmology," Phys. Rev. D 76, 103516 (2007).

${ }^{155} \mathrm{M}$. Cruz, N. Cruz, and S. Lepe, "Accelerated and decelerated expansion in a causal dissipative cosmology,” Phys. Rev. D 96, 124020 (2017).

${ }^{156} \mathrm{~B}$. Li and J. D. Barrow, "Does bulk viscosity create a viable unified dark matter model?," Phys. Rev. D 79, 103521 (2009).

${ }^{157}$ S. R. de Groot and P. Mazur, Non-Equilibrium Thermodynamics (Dover, 1962). ${ }^{158}$ D. Jou, J. Casas-Vázquez, and G. Lebon, Extended Irreversible Thermodynamics (Springer, 2010).

${ }^{159}$ P. Borgelt, C. Hoheisel, and G. Stell, "Exact molecular dynamics and kinetic theory results for thermal transport coefficients of the Lennard-Jones argon fluid in a wide range of states," Phys. Rev. A 42, 789 (1990).

${ }^{160} \mathrm{~K}$. Tankeshwar, "Bulk viscosity and the relation between transport coefficients,” Phys. Chem. Liq. 24, 91 (1991). 